\title{
Singular Braids and Partial Permutations
}

\author{
James East* \\ School of Mathematics and Statistics, University of Sydney, New South Wales 2006, Australia
}

January 14, 2010

\begin{abstract}
We introduce and study the partial singular braid monoid $\mathcal{P S B}_{n}$, a monoid that contains both the inverse braid monoid $\mathcal{I} \mathcal{B}_{n}$ and the singular braid monoid $\mathcal{S B}_{n}$. Our main results include a characterization of Green's relations, a presentation in terms of generators and relations, and a proof that $\mathcal{P} \mathcal{S B}_{n}$ embeds in the semigroup algebra $\mathbb{C}\left[\mathcal{I} \mathcal{B}_{n}\right]$.

Keywords: Braids, Singular braid monoids, Inverse braid monoids, Presentations, Birman's conjecture.
\end{abstract}

MSC: 20F36; 20M05.

\section{Introduction}

Many knot and link invariants are defined in terms of recursive formulae which allow reduction to simpler links. Links can be simplified in a number of ways, including cutting strings, or allowing certain strings to pass through each other. Just as the closure operator on links (Alexander's Theorem [1]) led to the introduction of the braid groups [3], so too do these link simplification operations lead to new classes of algebras; examples include the inverse braid monoids $\mathcal{I B}_{n}[12]$, singular braid monoids $\mathcal{S B}_{n}[5,7]$, factorizable braid monoids $\mathfrak{F B}_{n}[11,15]$, and permeable braid monoids $\mathfrak{P B}_{n}$ [18]. Some of these monoids may also be thought of as "braid analogues" of various semigroups of transformations that extend the symmetric groups; for other examples of such monoids, see [17, 25]. It is the purpose of this article to introduce and study the partial singular braid monoid $\mathcal{P} \mathcal{S B}_{n}$. This monoid simultaneously extends $\mathcal{I B}_{n}$ and $\mathcal{S B}_{n}$, and may also be thought of as a "singular braid analogue" of the symmetric inverse monoid $\mathcal{I}_{n}$ (the inverse monoid of all partial permutations of $\{1, \ldots, n\}$, under the operation of composition as binary relations), or a "partialized version" of $\mathcal{S B}_{n}$. The elements of $\mathcal{P} \mathcal{S} \mathcal{B}_{n}$ are essentially singular braids with

*james. east @ sydney.edu.au 
some (possibly none, or all) strings removed, and the multiplication operation is a natural extension of that for partial braids [12]. It is possibly not surprizing that the notion of partiality here is related to the "restriction categories" of [8]; the category whose objects are the finite subsets of $\mathbb{Z}^{+}$, with $\operatorname{Hom}(A, B)$ being equal to the set of all partial singular braids $\beta$ whose corresponding partial permutation $\bar{\beta}$ has $\operatorname{dom}(\bar{\beta}) \subseteq A$ and $\operatorname{im}(\bar{\beta}) \subseteq B$, is a restriction category - the idempotent associated to a morphism $\beta \in \operatorname{Hom}(A, B)$ is the idempotent partial braid whose associated partial permutation is the identity map on $A$ (we think of partial permutations as acting from the right, so the definitions from [8] need to be modified to fit in with this convention). It should be noted that $\mathcal{P} \mathcal{S B}_{n}$ is not the first generalization of $\mathcal{S B}_{n}$, as there is a large and growing literature on the singular Artin monoids introduced by Corran in [9]. One of the main themes for study in singular Artin monoids is the so-called Generalized Birman Conjecture, which concerns the injectivity of a certain map from the singular Artin monoid to the group algebra of its corresponding Artin group; see for example [2, 13, 21], and also [7] for the original formulation of the conjecture, [27] for its proof, and [26] for its generalization to singular braid monoids on closed surfaces.

This article is organized as follows. In Section 2 we define the partial singular braid monoid $\mathcal{P} \mathcal{S B}_{n}$, detailing some aspects of its structure, and culminating in a set of generators. In Section 3 we characterize Green's relations on $\mathcal{P S B}_{n}$, and also deduce characterizations for $\mathcal{I B}_{n}$ and $\mathcal{S B}_{n}$. We then explore more of the structure of $\mathcal{P} \mathcal{S B}_{n}$ when we study the pure partial singular braid monoid $\mathcal{P S} \mathcal{P}_{n}$ in Section 4 and then, in Section 5 , we find a system of defining relations for $\mathcal{P} \mathcal{S} \mathcal{B}_{n}$ with respect to the generating set alluded to above. Finally, in Section 6 we prove an analogue of Birman's Conjecture; specifically, we show that $\mathcal{P} \mathcal{S B}_{n}$ may be embedded in the complex semigroup algebra of the inverse braid monoid $\mathcal{I} \mathcal{B}_{n}$.

\section{The Partial Singular Braid Monoid}

Let $n$ be a positive integer, which we will fix for the remainder of the article, and write $\mathbf{n}=\{1, \ldots, n\}$. A partial singular braid on $\mathbf{n}$ is defined to be an object $\beta$ obtained by removing some (possibly all, or none) of the strings from a singular braid $\gamma$. (The reader is referred to [7] for details concerning the singular braid monoid $\mathcal{S B}_{n}$; see also [5].) In this case we say that $\beta$ is a sub-braid of $\gamma$, and we write $\beta \subseteq \gamma$. See Figure 1 for an illustration.

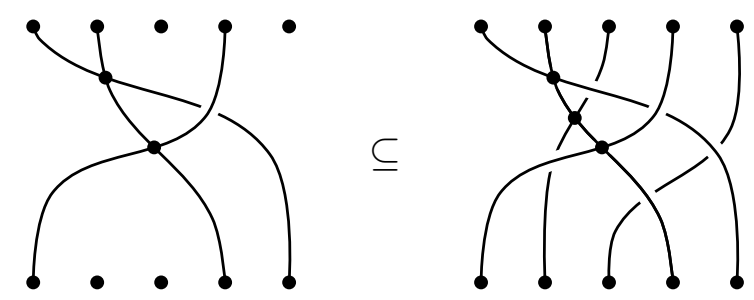

Figure 1: A sub-braid $\beta$ (left) of a singular braid $\gamma$ (right). 
A partial singular braid $\beta$ induces a partial permutation $\bar{\beta} \in \mathcal{I}_{n}$ in a natural way; the domain (resp. image) of $\bar{\beta}$ corresponds to the initial (resp. terminal) points of the strings of $\beta$ and, for $i \in \operatorname{dom}(\bar{\beta})$ and $j \in \operatorname{im}(\bar{\beta})$, we have $i \bar{\beta}=j$ if and only if a string of $\beta$ connects upper point $i$ to lower point $j$. So, with $\beta$ as in Figure 1 , we have $\bar{\beta}=\left(\begin{array}{ccccc}1 & 2 & 3 & 4 & 5 \\ 5 & 4 & - & 1 & -\end{array}\right)$, using an obvious tableaux notation for the elements of $\mathcal{I}_{n}$. If $\beta$ is a sub-braid of a singular braid $\gamma$ and $\operatorname{dom}(\bar{\beta})=A$, then we write $\beta=\left.\gamma\right|_{A}$ and call $\beta$ the restriction of $\gamma$ to $A$.

We say that two partial singular braids $\beta_{1}$ and $\beta_{2}$ are equivalent, and write $\beta_{1} \equiv \beta_{2}$, if

(i) $\bar{\beta}_{1}=\bar{\beta}_{2}$; and

(ii) $\beta_{1} \subseteq \gamma_{1}$ and $\beta_{2} \subseteq \gamma_{2}$ for some singular braids $\gamma_{1}$ and $\gamma_{2}$ which are equivalent in the usual sense of rigid-vertex-isotopy [7].

Given two partial singular braids $\beta$ and $\gamma$, the product $\beta \gamma$ is formed by first concatenating $\beta$ and $\gamma$ in the usual way, and then removing any string fragments which do not join an upper point to a lower point. See Figure 2 for an illustration. It is easy to see that $\beta_{1} \equiv \beta_{2}$

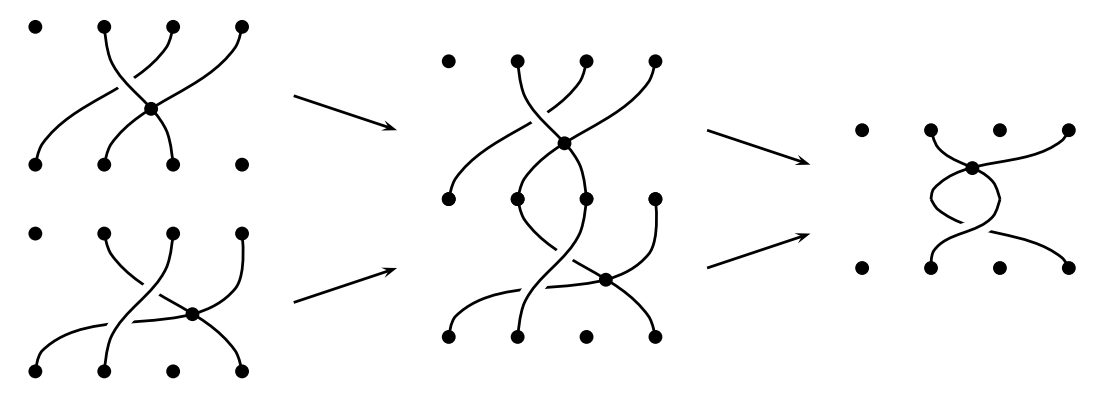

Figure 2: Multiplication of partial singular braids.

and $\gamma_{1} \equiv \gamma_{2}$ together imply $\beta_{1} \gamma_{1} \equiv \beta_{2} \gamma_{2}$, and that the induced product on $\equiv$-classes is accociative and unital (the identity is the $\equiv$-class of the braid with $n$ vertical strings). So the set $\mathcal{P} \mathcal{S B}_{n}$ of all $\equiv$-classes of partial singular braids on $\mathbf{n}$ is a monoid, which we call the partial singular braid monoid on $\mathbf{n}$. In practice, we will blur the distinction between a partial singular braid and its 三-class, but this should never cause any confusion.

If $\beta \in \mathcal{P} \mathcal{S B}_{n}$, we will write

- $|\beta|$ for the number of strings of $\beta$; and

- $N(\beta)$ for the number of singular points of $\beta$.

Note that also $|\beta|=\operatorname{rank}(\bar{\beta})=|\operatorname{dom}(\bar{\beta})|=|\operatorname{im}(\bar{\beta})|$. It is also immediate from the definitions that

$$
|\beta \gamma| \leq \min (|\beta|,|\gamma|) \quad \text { and } \quad N(\beta \gamma) \leq N(\beta)+N(\gamma)
$$

for all $\beta, \gamma \in \mathcal{P} \mathcal{S B}_{n}$. These definitions allow convenient description of the submonoids 
- $\mathcal{I B}_{n}=\left\{\beta \in \mathcal{P S B}_{n} \mid N(\beta)=0\right\} ;$ and

- $\mathcal{S B}_{n}=\left\{\beta \in \mathcal{P} \mathcal{S B}_{n}|| \beta \mid=n\right\}$.

As mentioned in Section 1, the monoid $\mathcal{I B}_{n}$ is known as the inverse braid monoid, and its elements are (equivalence classes of) partial braids; see [12]. As always, $\mathcal{S B}_{n}$ denotes the singular braid monoid $[5,7]$. The intersection of $\mathcal{I B}_{n}$ and $\mathcal{S B}_{n}$ is the braid group $\mathcal{B}_{n}$, which is the group of units of $\mathcal{P} \mathcal{S} \mathcal{B}_{n}$.

For $A \subseteq \mathbf{n}$, write $\varepsilon_{A}=\left.1\right|_{A}$ for the restriction of the identity braid $1 \in \mathcal{B}_{n}$ to $A$. Note that $\varepsilon_{A}$ is idempotent, and that $\left.\beta\right|_{A}=\varepsilon_{A} \beta$ for all $\beta \in \mathcal{S} \mathcal{B}_{n}$. It is also immediate that $\varepsilon_{A} \varepsilon_{B}=\varepsilon_{A \cap B}=\varepsilon_{B} \varepsilon_{A}$ for all $A, B$, so the set $\mathcal{E}_{n}=\left\{\varepsilon_{A} \mid A \subseteq \mathbf{n}\right\}$ is a semilattice isomorphic to the power-set $\left(2^{\mathbf{n}}, \cap\right)$.

Proposition 1 The semilattice $\mathcal{E}_{n}$ is equal to the set $E\left(\mathcal{P} \mathcal{S} \mathcal{B}_{n}\right)$ of all idempotents of $\mathcal{P} \mathcal{S}_{n}$. Further, we have

$$
\mathcal{P S} \mathcal{B}_{n}=\mathcal{E}_{n} \mathcal{S B}_{n}=\mathcal{S B}_{n} \mathcal{E}_{n}
$$

Proof Since every element of $\mathcal{E}_{n}$ is idempotent, we have $\mathcal{E}_{n} \subseteq E\left(\mathcal{P} \mathcal{S} \mathcal{B}_{n}\right)$. To prove the reverse inclusion, suppose $\beta \in E\left(\mathcal{P S B}_{n}\right)$ and put $A=\operatorname{dom}(\bar{\beta})$. Since $|\beta|=\left|\beta^{2}\right|$, we see that $\operatorname{im}(\bar{\beta})=A$ also, and so $\beta$ lies in an isomorphic copy of $\mathcal{S B}_{|A|}$. But then $N(\beta)=N\left(\beta^{2}\right)=N(\beta)+N(\beta)$ which forces $N(\beta)=0$, so that $\beta \in \mathcal{I B}_{n}$. We have shown that $E\left(\mathcal{P} \mathcal{S B}_{n}\right)=E\left(\mathcal{I B}_{n}\right)$ so the first claim follows from [12, Theorem 1.2].

For the final statement, if $\beta \in \mathcal{P} \mathcal{S B}_{n}$ with $\operatorname{dom}(\bar{\beta})=A$ and $\operatorname{im}(\bar{\beta})=B$, then we clearly have $\beta=\varepsilon_{A} \gamma=\gamma \varepsilon_{B}$ for any singular braid $\gamma \in \mathcal{S B}_{n}$ that contains $\beta$.

For $1 \leq i \leq n-1$, denote by $\sigma_{i}^{ \pm 1}$ and $\tau_{i}$ the standard singular braid generators [5, 7] and, for $1 \leq r \leq n$, put $\varepsilon_{r}=\varepsilon_{\mathbf{n} \backslash\{r\}}$ as in [16, 20]; see Figure 3 for an illustration. It is clear that for any $A \subseteq \mathbf{n}$, we have $\varepsilon_{A}=\varepsilon_{i_{1}} \cdots \varepsilon_{i_{k}}$ where $\mathbf{n} \backslash A=\left\{i_{1}, \ldots, i_{k}\right\}$.

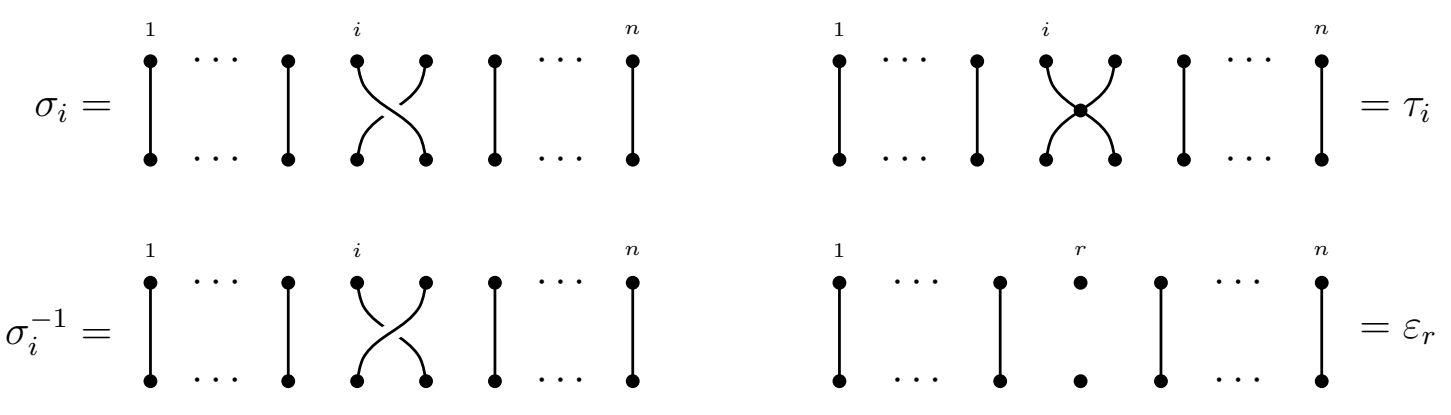

Figure 3: The generators of $\mathcal{P} \mathcal{S B}_{n}$.

Corollary 2 The partial singular braid monoid $\mathcal{P} \mathcal{S B}_{n}$ is generated by the set

$$
\Sigma_{n}=\left\{\sigma_{1}^{ \pm 1}, \ldots, \sigma_{n-1}^{ \pm 1}\right\} \cup\left\{\tau_{1}, \ldots, \tau_{n-1}\right\} \cup\left\{\varepsilon_{1}, \ldots, \varepsilon_{n}\right\} .
$$




\section{Green's Relations}

Green's relations $\mathscr{R}, \mathscr{L}, \mathscr{J}, \mathscr{H}, \mathscr{D}$ on a monoid $M$, introduced in the seminal paper [23], are defined in terms of mutual accessability by left and/or right multiplication. These relations encompass much of the structure of $M$, allowing its elements to be arranged neatly into rectangular arrays known as "egg boxes". Specifically, we have

- $x \mathscr{R} y \Longleftrightarrow x M=y M$

- $x \mathscr{L} y \Longleftrightarrow M x=M y$;

- $x \mathscr{J} y \Longleftrightarrow M x M=M y M$

- $\mathscr{H}=\mathscr{R} \cap \mathscr{L}$; and

- $\mathscr{D}=\mathscr{R} \vee \mathscr{L}=\mathscr{R} \circ \mathscr{L}=\mathscr{L} \circ \mathscr{R}$.

It is our goal in this section to characterize Green's relations in the partial singular braid monoid $\mathcal{P} \mathcal{S B}_{n}$. This characterization depends on various domains and images of the associated partial permutations, as well as a structural feature of $\mathcal{S B}_{n}$ discovered by Paris [27]. We begin with a lemma which describes Green's relations in terms of translations by units (braids).

Lemma 3 Let $\beta, \gamma \in \mathcal{P S} \mathcal{B}_{n}$. Then

(i) $\beta \mathscr{R} \gamma \Longleftrightarrow \beta=\gamma \delta$ for some $\delta \in \mathcal{B}_{n}$;

(ii) $\beta \mathscr{L} \gamma \Longleftrightarrow \beta=\delta \gamma$ for some $\delta \in \mathcal{B}_{n}$;

(iii) $\beta \mathscr{H} \gamma \Longleftrightarrow \beta=\gamma \delta_{1}=\delta_{2} \gamma$ for some $\delta_{1}, \delta_{2} \in \mathcal{B}_{n}$; and

(iv) $\beta \mathscr{J} \gamma \Longleftrightarrow \beta \mathscr{D} \gamma \Longleftrightarrow \beta=\delta_{1} \gamma \delta_{2}$ for some $\delta_{1}, \delta_{2} \in \mathcal{B}_{n}$.

Proof We first prove (i). Now $\beta=\gamma \delta$ with $\delta \in \mathcal{B}_{n}$ immediately implies $\beta \mathscr{R} \gamma$, since then also $\gamma=\beta \delta^{-1}$. For the converse, suppose $\beta \mathscr{R} \gamma$ and write $A=\operatorname{im}(\bar{\beta})$ and $B=\operatorname{im}(\bar{\gamma})$. Now

$$
\beta=\gamma \alpha_{1} \quad \text { and } \quad \gamma=\beta \alpha_{2}
$$

for some $\alpha_{1}, \alpha_{2} \in \mathcal{P} \mathcal{S B}_{n}$. Note first that $|\beta|=|\gamma|$ since $|\beta|=\left|\gamma \alpha_{1}\right| \leq|\gamma|$ and similarly $|\gamma| \leq|\beta|$. Now put $\alpha_{3}=\alpha_{1} \varepsilon_{A}$. Then since $\operatorname{im}\left(\bar{\alpha}_{1}\right) \supseteq \operatorname{im}\left(\overline{\gamma \alpha_{1}}\right)=\operatorname{im}(\bar{\beta})=A$, it follows that $\operatorname{im}\left(\bar{\alpha}_{3}\right)=A$. We also have

$$
\beta=\beta \varepsilon_{A}=\gamma \alpha_{1} \varepsilon_{A}=\gamma \alpha_{3}
$$

Since $|\beta|=|\gamma|=\left|\alpha_{3}\right|$, it follows that

$$
N(\beta)=N(\gamma)+N\left(\alpha_{3}\right)
$$


By symmetry, we also have $\gamma=\beta \alpha_{4}$ for some $\alpha_{4} \in \mathcal{P} \mathcal{S B}_{n}$ with $\operatorname{im}\left(\bar{\alpha}_{4}\right)=B$ and

$$
N(\gamma)=N(\beta)+N\left(\alpha_{4}\right)
$$

These two equations together imply that $N\left(\alpha_{3}\right)=N\left(\alpha_{4}\right)=0$. Now choose any two braids $\delta_{1}, \delta_{2} \in \mathcal{B}_{n}$ such that $\alpha_{3} \subseteq \delta_{1}$ and $\alpha_{4} \subseteq \delta_{2}$. Then by construction we have

$$
\beta=\gamma \alpha_{3}=\gamma \delta_{1} \quad \text { and } \quad \gamma=\beta \alpha_{4}=\beta \delta_{2} .
$$

This completes the proof of (i).

Part (ii) follows by a dual argument, while (iii) is an immediate consequence of (i) and (ii). For (iv), $\mathscr{D} \subseteq \mathscr{J}$ holds in any semigroup, while $\beta=\delta_{1} \gamma \delta_{2}$ for some $\delta_{1}, \delta_{2} \in \mathcal{B}_{n}$ implies that $\beta \mathscr{D} \gamma$ since then $\beta \mathscr{R} \beta \delta_{2} \mathscr{L} \gamma$. Finally, we modify the argument from part (i) above to show that $\beta \mathscr{J} \gamma$ implies $\beta=\delta_{1} \gamma \delta_{2}$ for some $\delta_{1}, \delta_{2} \in \mathcal{B}_{n}$.

If $A, B \subseteq \mathbf{n}$ are sets of the same cardinality, we write $\pi_{A, B} \in \mathcal{I B}_{n}$ for the partial braid with no crossings that satisfies $\operatorname{dom}\left(\bar{\pi}_{A, B}\right)=A$ and $\operatorname{im}\left(\bar{\pi}_{A, B}\right)=B$. See Figure 4 for an example.

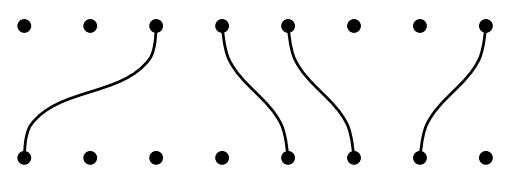

Figure 4: The partial braid $\pi_{A, B} \in \mathcal{I B}_{8}$, where $A=\{3,4,5,8\}$ and $B=\{1,5,6,7\}$.

The set $\left\{\pi_{A, B} \mid A, B \subseteq \mathbf{n}\right.$ and $\left.|A|=|B|\right\}$ is a (finite) submonoid of $\mathcal{P} \mathcal{S B}_{n}$ and is isomorphic to $\mathcal{P O} \mathcal{I}_{n}$, the monoid of all order-preserving partial permutations on $\mathbf{n}$; see [14, 19]. If $A \subseteq \mathbf{n}$ with $|A|=k$, we write

$$
\lambda_{A}=\pi_{A, \mathbf{k}} \quad \text { and } \quad \rho_{A}=\pi_{\mathbf{k}, A} .
$$

From the rule $\pi_{A, B} \pi_{B, C}=\pi_{A, C}$, we immediately obtain

$$
\lambda_{A} \rho_{B}=\pi_{A, B}, \quad \lambda_{A} \rho_{A}=\varepsilon_{A}, \quad \rho_{A} \lambda_{A}=\varepsilon_{\mathbf{k}} .
$$

If $p$ and $q$ are non-negative integers with $p \leq q$, then it is immediate from the definitions that any partial singular braid on $\mathbf{p}$ is also a partial singular braid on $\mathbf{q}$. In this way, the singular braid monoids $\mathcal{S B}_{k}(k=0,1, \ldots, n)$ are all contained in $\mathcal{P} \mathcal{S B}_{n}$. (Here we view $\mathcal{S B}_{0}$ as the singleton monoid consisting only of the empty braid.) Specifically, we have

$$
\mathcal{S B}_{k}=\left\{\beta \in \mathcal{P} \mathcal{S B}_{n} \mid \operatorname{dom}(\bar{\beta})=\operatorname{im}(\bar{\beta})=\mathbf{k}\right\}
$$

for each $k$. The next lemma will prove useful on a number of occasions.

Lemma 4 Let $\beta \in \mathcal{P S B}_{n}$ and put $A=\operatorname{dom}(\bar{\beta}), B=\operatorname{im}(\bar{\beta})$, and $k=|\beta|$. Then $\widehat{\beta}=\rho_{A} \beta \lambda_{B} \in \mathcal{S B}_{k}$ is the unique singular braid on $\mathbf{k}$ for which $\beta=\lambda_{A} \widehat{\beta} \rho_{B}$. 
Proof Since $\operatorname{im}\left(\bar{\rho}_{A}\right)=A=\operatorname{dom}(\bar{\beta})$, and $\operatorname{im}(\bar{\beta})=B=\operatorname{dom}\left(\bar{\lambda}_{B}\right)$, we see that $|\widehat{\beta}|=|\beta|=k$, and we immediately deduce that $\widehat{\beta} \in \mathcal{S B}_{k}$. We also have

$$
\lambda_{A} \widehat{\beta} \rho_{B}=\lambda_{A} \rho_{A} \beta \lambda_{B} \rho_{B}=\varepsilon_{A} \beta \varepsilon_{B}=\beta .
$$

Finally, if $\gamma \in \mathcal{S B}_{k}$ also satisfies $\beta=\lambda_{A} \gamma \rho_{B}$, then

$$
\gamma=\varepsilon_{\mathbf{k}} \gamma \varepsilon_{\mathbf{k}}=\rho_{A} \lambda_{A} \gamma \rho_{B} \lambda_{B}=\rho_{A} \beta \lambda_{B}=\widehat{\beta}
$$

This completes the proof.

This structure will allow us to characterize Green's relations in a more computable way. With this in mind, we first recall some structural results from [27] concerning the singular braid monoid $\mathcal{S B}_{n}$. For $1 \leq i \leq n-1$ and $\beta \in \mathcal{B}_{n}$ we define a singular braid $\gamma_{i, \beta}=\beta^{-1} \sigma_{i} \tau_{i} \beta \in \mathcal{S B}_{n}$ and put

$$
\Gamma_{n}=\left\{\gamma_{i, \beta} \mid 1 \leq i \leq n-1, \beta \in \mathcal{B}_{n}\right\}
$$

We write $\mathcal{G}_{n}=\left\langle\Gamma_{n}\right\rangle$ for the submonoid of $\mathcal{S B}_{n}$ generated by $\Gamma_{n}$. By [27, Lemma 2.3], we have the internal semidirect product decompositions

$$
\mathcal{S B}_{n}=\mathcal{G}_{n} \rtimes \mathcal{B}_{n}=\mathcal{B}_{n} \ltimes \mathcal{G}_{n}
$$

(Further information is given in [27] concerning the structure of $\mathcal{G}_{n}$ as a graph monoid on the vertext set $\Gamma_{n}$, but we do not need it here.) So, for any $\beta \in \mathcal{S B}_{n}$, we have

$$
\beta=L_{G}(\beta) R_{B}(\beta)=L_{B}(\beta) R_{G}(\beta)
$$

for unique $L_{G}(\beta), R_{G}(\beta) \in \mathcal{G}_{n}$ and $L_{B}(\beta), R_{B}(\beta) \in \mathcal{B}_{n}$. In fact, we always have

$$
R_{B}(\beta)=L_{B}(\beta) \quad \text { and } \quad R_{G}(\beta)=L_{B}(\beta)^{-1} L_{G}(\beta) L_{B}(\beta),
$$

but it will still be helpful to refer to the "four" maps $L_{G}, R_{G}, L_{B}, R_{B}$. Note also that if $\beta \in \mathcal{S B}_{n}$ and $\gamma \in \mathcal{B}_{n}$, then

$$
\begin{aligned}
& L_{G}(\beta \gamma)=L_{G}(\beta), \quad R_{B}(\beta \gamma)=R_{B}(\beta) \gamma, \\
& L_{B}(\gamma \beta)=\gamma L_{B}(\beta), \quad R_{G}(\gamma \beta)=R_{G}(\beta) .
\end{aligned}
$$

For $0 \leq k \leq n$, we will write $L_{G ; k}$ etc. for the corresponding maps on $\mathcal{S B}_{k}$.

Theorem 5 Let $\beta, \gamma \in \mathcal{P S} \mathcal{B}_{n}$ and put $k=|\beta|$. Then

(i) $\beta \mathscr{R} \gamma \Longleftrightarrow \operatorname{dom}(\bar{\beta})=\operatorname{dom}(\bar{\gamma})$ and $L_{G ; k}(\widehat{\beta})=L_{G ; k}(\widehat{\gamma})$;

(ii) $\beta \mathscr{L} \gamma \Longleftrightarrow \operatorname{im}(\bar{\beta})=\operatorname{im}(\bar{\gamma})$ and $R_{G ; k}(\widehat{\beta})=R_{G ; k}(\widehat{\gamma})$;

(iii) $\beta \mathscr{H} \gamma \Longleftrightarrow \operatorname{dom}(\bar{\beta})=\operatorname{dom}(\bar{\gamma}), \operatorname{im}(\bar{\beta})=\operatorname{im}(\bar{\gamma}), L_{G ; k}(\widehat{\beta})=L_{G ; k}(\widehat{\gamma})$, and $R_{G ; k}(\widehat{\beta})=$ $R_{G ; k}(\widehat{\gamma}) ;$ and 
(iv) $\beta \mathscr{J} \gamma \Longleftrightarrow \beta \mathscr{D} \gamma \Longleftrightarrow|\gamma|=k$ and $L_{G ; k}(\widehat{\beta}), R_{G ; k}(\widehat{\beta}), L_{G ; k}(\widehat{\gamma}), R_{G ; k}(\widehat{\gamma})$ are all conjugate via braids (from $\mathcal{B}_{k}$ ).

Proof We start with part (i). Suppose first that $\operatorname{dom}(\bar{\beta})=\operatorname{dom}(\bar{\gamma})$ and $L_{G ; k}(\widehat{\beta})=L_{G ; k}(\widehat{\gamma})$. Put

$$
A=\operatorname{dom}(\bar{\beta})=\operatorname{dom}(\bar{\gamma}), \quad B=\operatorname{im}(\bar{\beta}), \quad C=\operatorname{im}(\bar{\gamma})
$$

Now

$$
\begin{aligned}
\beta & =\lambda_{A} \widehat{\beta} \rho_{B} \\
& =\lambda_{A} L_{G ; k}(\widehat{\beta}) R_{G ; k}(\widehat{\beta}) \rho_{B} \\
& =\lambda_{A} L_{G ; k}(\widehat{\gamma}) R_{B ; k}(\widehat{\gamma})\left(R_{B ; k}(\widehat{\gamma})\right)^{-1} R_{B ; k}(\widehat{\beta}) \rho_{B} \\
& =\lambda_{A} \widehat{\gamma} \varepsilon_{\mathbf{k}}\left(R_{B ; k}(\widehat{\gamma})\right)^{-1} R_{B ; k}(\widehat{\beta}) \rho_{B} \\
& =\lambda_{A} \widehat{\gamma} \rho_{C} \lambda_{C}\left(R_{B ; k}(\widehat{\gamma})\right)^{-1} R_{B ; k}(\widehat{\beta}) \rho_{B} \\
& =\gamma \alpha,
\end{aligned}
$$

where $\alpha=\lambda_{C}\left(R_{B ; k}(\widehat{\gamma})\right)^{-1} R_{B ; k}(\widehat{\beta}) \rho_{B}$, and throughout $\left(R_{B ; k}(\widehat{\gamma})\right)^{-1}$ denotes the inverse with respect to $\varepsilon_{\mathbf{k}}$, the identity of $\mathcal{B}_{k}$. Since $\alpha \in \mathcal{I B}_{n}$, we have $\alpha \subseteq \delta$ for some braid $\delta \in \mathcal{B}_{n}$ and, since $\operatorname{dom}(\bar{\alpha})=C=\operatorname{im}(\bar{\gamma})$, it follows that $\beta=\gamma \alpha=\gamma \delta$ so that $\beta \mathscr{R} \gamma$ by Lemma 3(i).

Conversely, suppose $\beta \mathscr{R} \gamma$. Then $\beta=\gamma \delta$ for some $\delta \in \mathcal{B}_{n}$ by Lemma 3(i). Now $\operatorname{dom}(\bar{\gamma})=$ $\operatorname{dom}(\overline{\gamma \delta})=\operatorname{dom}(\bar{\beta})$ since $\bar{\delta}$ is a permutation. Write

$$
A=\operatorname{dom}(\bar{\beta})=\operatorname{dom}(\bar{\gamma}), \quad B=\operatorname{im}(\bar{\beta}), \quad C=\operatorname{im}(\bar{\gamma}) .
$$

Note first that $C \bar{\delta}=\operatorname{im}(\bar{\gamma}) \bar{\delta}=\operatorname{im}(\bar{\gamma} \bar{\delta})=\operatorname{im}(\bar{\beta})=B$ since $\bar{\delta}$ is a permutation. We also have

$$
\beta=\beta \varepsilon_{B}=\gamma \delta \varepsilon_{B}=\lambda_{A}\left(\widehat{\gamma} \rho_{C} \delta \lambda_{B}\right) \rho_{B}
$$

But $\rho_{C} \delta \lambda_{B} \in \mathcal{B}_{k}$ since $\delta \in \mathcal{B}_{n}$ and $C \bar{\delta}=B$, so it follows that $\widehat{\gamma} \rho_{C} \delta \lambda_{B} \in \mathcal{S B}_{k}$. But then Lemma 4 implies that $\widehat{\gamma} \rho_{C} \delta \lambda_{B}=\widehat{\beta}$, and it follows that

$$
L_{G ; k}(\widehat{\gamma})=L_{G ; k}\left(\widehat{\gamma} \rho_{C} \delta \lambda_{B}\right)=L_{G ; k}(\widehat{\beta}) .
$$

This completes the proof of (i).

Again, (ii) follows from a dual argument, and (iii) is an immediate consequence of (i) and (ii).

For (iv), we have already established in Lemma 3 that $\mathscr{J}=\mathscr{D}$. Put

$$
A=\operatorname{dom}(\bar{\beta}), \quad B=\operatorname{im}(\bar{\beta}), \quad C=\operatorname{dom}(\bar{\gamma}), \quad D=\operatorname{im}(\bar{\gamma}) .
$$

Suppose first that $\beta \mathscr{J} \gamma$. Then $\beta=\delta_{1} \gamma \delta_{2}$ for some $\delta_{1}, \delta_{2} \in \mathcal{B}_{n}$, by Lemma 3(iv), and it follows that $|\gamma|=|\beta|=k$. Now

$$
\widehat{\beta}=\rho_{A} \beta \lambda_{B}=\rho_{A} \delta_{1} \gamma \delta_{2} \lambda_{B}=\rho_{A} \delta_{1} \lambda_{C} \widehat{\gamma} \rho_{D} \delta_{2} \lambda_{B}=\alpha_{1} \widehat{\gamma} \alpha_{2},
$$


where $\alpha_{1}=\rho_{A} \delta_{1} \lambda_{C}, \alpha_{2}=\rho_{D} \delta_{2} \lambda_{B} \in \mathcal{B}_{k}$. We then have

$$
L_{G ; k}(\widehat{\beta}) R_{B ; k}(\widehat{\beta})=\widehat{\beta}=\alpha_{1} \widehat{\gamma} \alpha_{2}=\alpha_{1} L_{G ; k}(\widehat{\gamma}) R_{B ; k}(\widehat{\gamma}) \alpha_{2}=\left[\alpha_{1} L_{G ; k}(\widehat{\gamma}) \alpha_{1}^{-1}\right] \alpha_{1} R_{B ; k}(\widehat{\gamma}) \alpha_{2} .
$$

(Again, $\alpha_{1}^{-1}$ denotes the inverse in $\mathcal{B}_{k}$.) Since $\alpha_{1} L_{G ; k}(\widehat{\gamma}) \alpha_{1}^{-1} \in \mathcal{G}_{k}$ and $\alpha_{1} R_{B ; k}(\widehat{\gamma}) \alpha_{2} \in \mathcal{B}_{k}$, it follows by [27, Lemma 2.3] that $L_{G ; k}(\widehat{\beta})=\alpha_{1} L_{G ; k}(\widehat{\gamma}) \alpha_{1}^{-1}$. The forwards implication is now proved, since $L_{G ; k}(\mu)$ and $R_{G ; k}(\mu)$ are always conjugate via braids for any $\mu \in \mathcal{S B}_{k}$, as we noted before the statement of the theorem.

For the reverse implication, suppose $|\beta|=|\gamma|=k$ and

$$
L_{G ; k}(\widehat{\beta})=\alpha L_{G ; k}(\widehat{\gamma}) \alpha^{-1}
$$

where $\alpha \in \mathcal{B}_{k}$ and $\alpha^{-1}$ denotes the inverse in $\mathcal{B}_{k}$. Then

$$
\begin{aligned}
\beta & =\lambda_{A} \widehat{\beta} \rho_{B} \\
& =\lambda_{A} L_{G ; k}(\widehat{\beta}) R_{B ; k}(\widehat{\beta}) \rho_{B} \\
& =\lambda_{A} \alpha L_{G ; k}(\widehat{\gamma}) \alpha^{-1} R_{B ; k}(\widehat{\beta}) \rho_{B} \\
& =\lambda_{A} \alpha L_{G ; k}(\widehat{\gamma}) R_{B ; k}(\widehat{\gamma})\left(R_{B ; k}(\widehat{\gamma})\right)^{-1} \alpha^{-1} R_{B ; k}(\widehat{\beta}) \rho_{B} \\
& =\lambda_{A} \alpha \varepsilon_{\mathbf{k}} \widehat{\gamma} \varepsilon_{\mathbf{k}}\left(R_{B ; k}(\widehat{\gamma})\right)^{-1} \alpha^{-1} R_{B ; k}(\widehat{\beta}) \rho_{B} \\
& =\lambda_{A} \alpha \rho_{C} \lambda_{C} \widehat{\gamma} \rho_{D} \lambda_{D}\left(R_{B ; k}(\widehat{\gamma})\right)^{-1} \alpha^{-1} R_{B ; k}(\widehat{\beta}) \rho_{B} \\
& =\alpha_{1} \gamma \alpha_{2},
\end{aligned}
$$

where $\alpha_{1}=\lambda_{A} \alpha \rho_{C}$ and $\alpha_{2}=\lambda_{D}\left(R_{B ; k}(\widehat{\gamma})\right)^{-1} \alpha^{-1} R_{B ; k}(\widehat{\beta}) \rho_{B}$. Now $\alpha_{1}, \alpha_{2} \in \mathcal{I} \mathcal{B}_{n}$, so we choose any braids $\delta_{1}, \delta_{2} \in \mathcal{B}_{n}$ such that $\alpha_{1} \subseteq \delta_{1}$ and $\alpha_{2} \subseteq \delta_{2}$. Since $\operatorname{im}\left(\bar{\alpha}_{1}\right)=C=\operatorname{dom}(\bar{\gamma})$ and $\operatorname{im}(\bar{\gamma})=D=\operatorname{dom}\left(\bar{\alpha}_{2}\right)$, we have

$$
\beta=\alpha_{1} \gamma \alpha_{2}=\delta_{1} \gamma \delta_{2}
$$

so that $\beta \mathscr{J} \gamma$ by Lemma 3(iv). This completes the proof.

Remark 6 Theorem 5 yields an algorithmic solution to the problem of determining whether two partial singular braids are equivalent under one of Green's relations. In each case, the problem breaks down into (i) the computation of various domains and/or images (this is trivial), (ii) the computations of various $L_{G ; k}$ and/or $R_{G ; k}$ factors (this is again trivial; see [21]), and (iii) a solution to the word problem [9] in the case of $\mathscr{R}, \mathscr{L}, \mathscr{H}$, or the conjugacy problem $[10]$ in the case of $\mathscr{D}=\mathscr{J}$.

Characterizations of Green's relations in $\mathcal{I B}_{n}$ and $\mathcal{S B}_{n}$ may be obtained directly, but to the author's knowledge are not stated anywhere in the literature. In any case, they are immediate consequences of Theorem 5 (and parts of its proof), so we state them here. 
Corollary 7 Let $\beta, \gamma \in \mathcal{I B}_{n}$. Then

(i) $\beta \mathscr{R} \gamma \Longleftrightarrow \operatorname{dom}(\bar{\beta})=\operatorname{dom}(\bar{\gamma})$;

(ii) $\beta \mathscr{L} \gamma \Longleftrightarrow \operatorname{im}(\bar{\beta})=\operatorname{im}(\bar{\gamma})$;

(iii) $\beta \mathscr{H} \gamma \Longleftrightarrow \operatorname{dom}(\bar{\beta})=\operatorname{dom}(\bar{\gamma})$ and $\operatorname{im}(\bar{\beta})=\operatorname{im}(\bar{\gamma})$; and

(iv) $\beta \mathscr{J} \gamma \Longleftrightarrow \beta \mathscr{D} \gamma \Longleftrightarrow|\beta|=|\gamma|$.

Corollary 8 Let $\beta, \gamma \in \mathcal{S B}_{n}$. Then

(i) $\beta \mathscr{R} \gamma \Longleftrightarrow L_{G}(\beta)=L_{G}(\gamma)$;

(ii) $\beta \mathscr{L} \gamma \Longleftrightarrow R_{G}(\beta)=R_{G}(\gamma)$;

(iii) $\beta \mathscr{H} \gamma \Longleftrightarrow L_{G}(\beta)=L_{G}(\gamma)$ and $R_{G}(\beta)=R_{G}(\gamma)$; and

(iv) $\beta \mathscr{J} \gamma \Longleftrightarrow \beta \mathscr{D} \gamma \Longleftrightarrow L_{G}(\beta), R_{G}(\beta), L_{G}(\gamma), R_{G}(\gamma)$ are all conjugate via braids.

\section{The Pure Partial Singular Braid Monoid}

It is immediate from the definitions that $\overline{\beta \gamma}=\bar{\beta} \bar{\gamma}$ for all $\beta, \gamma \in \mathcal{P} \mathcal{S} \mathcal{B}_{n}$, so we have an epimorphism $\mathcal{P S B}_{n} \rightarrow \mathcal{I}_{n}: \beta \mapsto \bar{\beta}$ which naturally extends the permutation map $\mathcal{S B}_{n} \rightarrow \mathcal{S}_{n}$. We define the pure partial singular braid monoid

$$
\mathcal{P S P}_{n}=\left\{\beta \in \mathcal{P} \mathcal{S B}_{n} \mid \bar{\beta}=\operatorname{id}_{A}(\exists A \subseteq \mathbf{n})\right\} .
$$

Note that $\mathcal{P S P} \mathcal{P}_{n}$ is the preimage under the above map of $E\left(\mathcal{I}_{n}\right)$, the semilattice of idempotents of $\mathcal{I}_{n}$. Key submonoids of $\mathcal{P} \mathcal{S P}{ }_{n}$ include

- $\mathcal{S P}_{n}=\mathcal{P} \mathcal{S P}_{n} \cap \mathcal{S B}_{n}$, the pure singular braid monoid [24];

- $\mathcal{I P}_{n}=\mathcal{P} \mathcal{S} \mathcal{P}_{n} \cap \mathcal{I B}_{n}$, the pure inverse braid monoid [16]; and

- $\mathcal{P}_{n}=\mathcal{P} \mathcal{S} \mathcal{P}_{n} \cap \mathcal{B}_{n}$, the pure braid group $[3,4]$.

The following diagram displays the various inclusions and intersections.

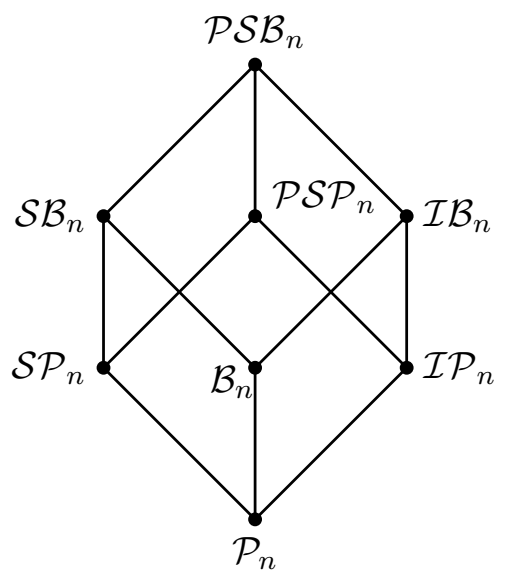


In this section we study the pure partial singular braid monoid $\mathcal{P} \mathcal{S} \mathcal{P}_{n}$, and record a number of structural results concerning generation. With this in mind, for $1 \leq i<j \leq n$, we define

$$
\begin{aligned}
\alpha_{i j} & =\left(\sigma_{j-1}^{-1} \cdots \sigma_{i+1}^{-1}\right) \sigma_{i}^{2}\left(\sigma_{i+1} \cdots \sigma_{j-1}\right) \in \mathcal{P}_{n}, \\
\gamma_{i j} & =\left(\sigma_{j-1}^{-1} \cdots \sigma_{i+1}^{-1}\right) \sigma_{i} \tau_{i}\left(\sigma_{i+1} \cdots \sigma_{j-1}\right) \in \mathcal{S P}_{n} .
\end{aligned}
$$

In the notation introduced after Lemma 4 , we have $\gamma_{i j}=\gamma_{i, \beta}$, where $\beta=\sigma_{i+1} \cdots \sigma_{j-1}$. See Figure 5 for an illustration.
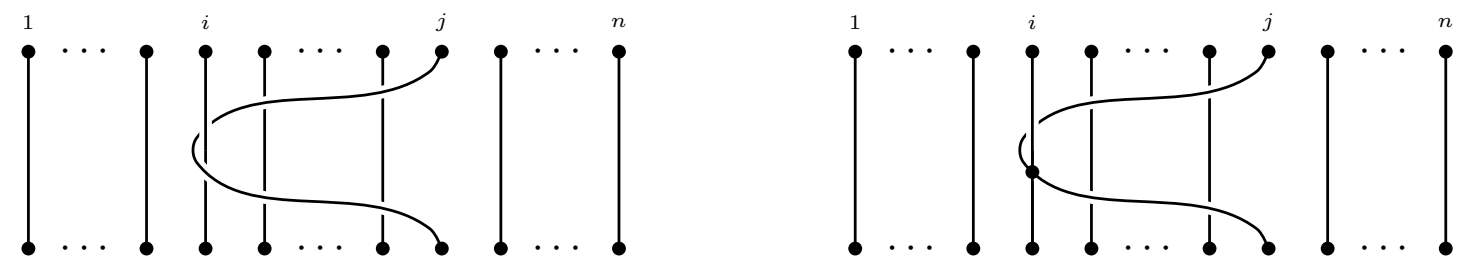

Figure 5: The pure braid $\alpha_{i j}$ (left) and pure singular braid $\gamma_{i j}$ (right).

It is a standard result that $\mathcal{P}_{n}$ is generated (as a group) by the $\alpha_{i j}$; see for example $[4,6]$. The next result has its origins in [24]; see also [13].

Proposition 9 The pure singular braid monoid $\mathcal{S P}_{n}$ is generated as a monoid by the set $\Upsilon_{n}=\left\{\alpha_{i j}^{ \pm 1}, \gamma_{i j} \mid 1 \leq i<j \leq n\right\}$.

Proof We clearly have $\Upsilon_{n} \subseteq \mathcal{S} \mathcal{P}_{n}$. Conversely, suppose $\beta \in \mathcal{S P}{ }_{n}$. Now $\beta=L_{G}(\beta) R_{B}(\beta)$ and, since $L_{G}(\beta) \in \mathcal{G}_{n} \subseteq \mathcal{S P}_{n}$, it follows that $R_{B}(\beta) \in \mathcal{P}_{n}=\left\langle\alpha_{i j}^{ \pm 1} \mid 1 \leq i<j \leq n\right\rangle$. So it suffices to show that the generators $\gamma_{i, \delta}=\delta^{-1} \sigma_{i} \tau_{i} \delta$ of $\mathcal{G}_{n}$ belong to $\left\langle\Upsilon_{n}\right\rangle$. But, since $\gamma_{i, \delta}=\delta^{-1} \gamma_{i, i+1} \delta$, so it is enough to show that $\delta^{-1} \gamma_{i j} \delta \in\left\langle\Upsilon_{n}\right\rangle$ for all $1 \leq i<j \leq n$ and $\delta \in \mathcal{B}_{n}$. Now one may easily check that

$$
\sigma_{k}^{-1} \gamma_{i j} \sigma_{k}= \begin{cases}\alpha_{i-1, i}^{-1} \gamma_{i-1, j} \alpha_{i-1, i} & \text { if } k=i-1 \\ \gamma_{i+1, j} & \text { if } k=i \neq j-1 \\ \alpha_{j-1, j}^{-1} \gamma_{i, j-1} \alpha_{j-1, j} & \text { if } k=j-1 \neq i \\ \gamma_{i, j+1} & \text { if } k=j \\ \gamma_{i j} & \text { otherwise }\end{cases}
$$

and

$$
\sigma_{k} \gamma_{i j} \sigma_{k}^{-1}= \begin{cases}\gamma_{i-1, j} & \text { if } k=i-1 \\ \alpha_{i, i+1} \gamma_{i+1, j} \alpha_{i, i+1}^{-1} & \text { if } k=i \neq j-1 \\ \gamma_{i, j-1} & \text { if } k=j-1 \neq i \\ \alpha_{j, j+1} \gamma_{i, j+1} \alpha_{j, j+1}^{-1} & \text { if } k=j \\ \gamma_{i j} & \text { otherwise. }\end{cases}
$$


The desired result now follows by a simple induction on $k$, where $\delta=\sigma_{i_{1}}^{ \pm 1} \cdots \sigma_{i_{k}}^{ \pm 1}$.

Remark 10 The author has not calculated a set of defining relations for $\mathcal{S P}_{n}$ with respect to the generating set $\Upsilon_{n}$. Relations may easily be obtained by first obtaining a presentation with respect to the infinite generating set $\Gamma_{n} \cup\left\{\alpha_{i j}^{ \pm 1} \mid 1 \leq i<j \leq n\right\}$, using the semidirect product structure of $\mathcal{S} \mathcal{P}_{n}=\mathcal{G}_{n} \rtimes \mathcal{P}_{n}$, and presentations for $\mathcal{G}_{n}$ (as a graph monoid) and $\mathcal{P}_{n}$, but the resulting presentation is infinite. The author suspects that $\mathcal{S P}_{n}$ is not finitely presented (although it is of course finitely generated).

For $A \subseteq \mathbf{n}$ we define

$$
\Upsilon_{A}=\left\{\alpha_{i j}^{ \pm 1}, \gamma_{i j} \mid 1 \leq i<j \leq n, i, j \in A\right\} .
$$

The next lemma is one of the key steps in establishing a presentation for $\mathcal{P S} \mathcal{B}_{n}$ in the next section.

Lemma 11 Let $\beta \in \mathcal{P S} \mathcal{P}_{n}$ and put $A=\operatorname{dom}(\bar{\beta})$. Then $\beta=\varepsilon_{A} \gamma$ for a unique $\gamma \in\left\langle\Upsilon_{A}\right\rangle$.

Proof We first prove existence. Now $\beta \subseteq \delta$ for some singular braid $\delta \in \mathcal{S B}_{n}$, and so $\beta=\left.\delta\right|_{A}=\varepsilon_{A} \delta$. Now for all $i \in A$ we have

$$
i \bar{\delta}=\left(i\left(\operatorname{id}_{A}\right)\right) \bar{\delta}=i \overline{\varepsilon_{A} \delta}=i \bar{\beta}=i
$$

since $\beta$ is pure. So we may write $\bar{\delta}=\left(i_{1}, j_{1}\right) \cdots\left(i_{k}, j_{k}\right)$ as a product of transpositions, where $1 \leq i_{r}<j_{r} \leq n$ and $i_{r}, j_{r} \in \mathbf{n} \backslash A$ for all $r \in \mathbf{k}$. Let $\alpha=\sigma_{i_{k} j_{k}} \cdots \sigma_{i_{1} j_{1}} \in \mathcal{B}_{n}$, where

$$
\sigma_{i j}=\left(\sigma_{j-1}^{-1} \cdots \sigma_{i+1}^{-1}\right) \sigma_{i}\left(\sigma_{i+1} \cdots \sigma_{j-1}\right) .
$$

Then $\varepsilon_{A} \alpha=\varepsilon_{A}$, as can easily be checked diagrammatically. But then, putting $\delta_{1}=\alpha \delta$, we have

$$
\beta=\varepsilon_{A} \delta=\varepsilon_{A} \alpha \delta=\varepsilon_{A} \delta_{1},
$$

and

$$
\bar{\delta}_{1}=\bar{\alpha} \bar{\delta}=\left(i_{k}, j_{k}\right) \cdots\left(i_{1}, j_{1}\right)\left(i_{1}, j_{1}\right) \cdots\left(i_{k}, j_{k}\right)=1 .
$$

Thus $\delta_{1} \in \mathcal{S P}_{n}=\left\langle\Upsilon_{n}\right\rangle$, so we have $\delta_{1}=\xi_{1} \cdots \xi_{\ell}$ for some $\xi_{1}, \ldots, \xi_{\ell} \in \Upsilon_{n}$. If all of the $\xi_{i}$ belong to $\Upsilon_{A}$ already, then we are done. Otherwise, if $i \in \boldsymbol{\ell}$ is minimal so that $\xi_{i} \in \Upsilon_{n} \backslash \Upsilon_{A}$, then we have $\varepsilon_{A} \xi_{i}=\varepsilon_{A}$, so that

$$
\begin{aligned}
\beta=\varepsilon_{A} \delta_{1} & =\varepsilon_{A} \xi_{1} \cdots \xi_{i-1} \xi_{i} \xi_{i+1} \cdots \xi_{\ell} \\
& =\xi_{1} \cdots \xi_{i-1} \varepsilon_{A} \xi_{i} \xi_{i+1} \cdots \xi_{\ell} \\
& =\xi_{1} \cdots \xi_{i-1} \varepsilon_{A} \xi_{i+1} \cdots \xi_{\ell} \\
& =\varepsilon_{A} \xi_{1} \cdots \xi_{i-1} \xi_{i+1} \cdots \xi_{\ell} .
\end{aligned}
$$


Since $\xi_{1} \cdots \xi_{i-1} \xi_{i+1} \cdots \xi_{\ell}$ has one fewer appearence of a generator from $\Upsilon_{n} \backslash \Upsilon_{A}$, we are done after applying an induction hypothesis.

To establish uniqueness, if $\beta=\varepsilon_{A} \gamma=\varepsilon_{A} \gamma^{\prime}$ where $\gamma, \gamma^{\prime} \in\left\langle\Upsilon_{A}\right\rangle$, then an isotopy from $\varepsilon_{A} \gamma$ to $\varepsilon_{A} \gamma^{\prime}$ in (an isomorphic copy of) $\mathcal{S} \mathcal{P}_{|A|}$ may be extended to give an isotopy from $\gamma$ to $\gamma^{\prime}$ in $\mathcal{S P}_{n}$ by adding the remaining $n-|A|$ strings (as straight strings passing behind the pre-existing strings) and keeping them out of the way during the isotopy.

\section{A Presentation}

It is our task in this section to find a presentation for $\mathcal{P S B}_{n}$ with respect to the generating set

$$
\Sigma_{n}=\left\{\sigma_{1}^{ \pm 1}, \ldots, \sigma_{n-1}^{ \pm 1}\right\} \cup\left\{\tau_{1}, \ldots, \tau_{n-1}\right\} \cup\left\{\varepsilon_{1}, \ldots, \varepsilon_{n}\right\}
$$

from Corollary 2. With this in mind, define alphabets

$$
S=\left\{s_{1}, \ldots, s_{n-1}\right\}, \quad S^{-1}=\left\{s_{1}^{-1}, \ldots, s_{n-1}^{-1}\right\}, \quad T=\left\{t_{1}, \ldots, t_{n-1}\right\}, \quad E=\left\{e_{1}, \ldots, e_{n}\right\},
$$

and put

$$
\mathscr{X}=S \cup S^{-1} \cup T \cup E .
$$

Since $\mathcal{P} \mathcal{S B}_{n}=\left\langle\Sigma_{n}\right\rangle$, we have an epimorphism

$$
\Phi: \mathscr{X}^{*} \rightarrow \mathcal{P} \mathcal{S B}_{n}: s_{i}^{ \pm 1} \mapsto \sigma_{i}^{ \pm 1}, t_{i} \mapsto \tau_{i}, e_{i} \mapsto \varepsilon_{i} .
$$

Consider the set $\mathscr{R}$ of relations

$$
\begin{array}{rlrl}
s_{i} s_{i}^{-1}=s_{i}^{-1} s_{i} & =1 & & \text { for all } i \\
s_{i} s_{j} & =s_{j} s_{i} & & \text { if }|i-j|>1 \\
s_{i} s_{j} s_{i} & =s_{j} s_{i} s_{j} & & \text { if }|i-j|=1 \\
t_{i} t_{j} & =t_{j} t_{i} & & \text { if }|i-j|>1 \\
s_{i} t_{j} & =t_{j} s_{i} & & \text { if }|i-j| \neq 1 \\
s_{i} s_{j} t_{i} & =t_{j} s_{i} s_{j} & & \text { if }|i-j|=1 \\
e_{i}^{2}=e_{i}=e_{i} s_{i}^{2} & =s_{i}^{2} e_{i} & \text { for all } i \\
e_{i} e_{j} & =e_{j} e_{i} & & \text { for all } i, j \\
s_{i} e_{j} & =e_{j} s_{i} & & \text { if } j \neq i, i+1 \\
e_{i} e_{i+1} s_{i} & =e_{i} e_{i+1} & \text { for all } i \\
t_{i} e_{j} & =e_{j} t_{i} & & \text { if } j \neq i, i+1 \\
t_{i} e_{i}=s_{i} e_{i}=e_{i+1} s_{i} & =e_{i+1} t_{i} & & \text { for all } i .
\end{array}
$$

It is our goal to show that $\mathcal{P} \mathcal{S} \mathcal{B}_{n} \cong\langle\mathscr{X} \mid \mathscr{R}\rangle$. From now on we denote by $\sim=\mathscr{R}^{\sharp}$ the congruence on $\mathscr{X}^{*}$ generated by $\mathscr{R}$. Since we know that $\Phi$ is an epimorphism, our task is to show that $\operatorname{ker} \Phi=\sim$. 
Lemma 12 We have the inclusion $\sim \subseteq \operatorname{ker} \Phi$.

Proof This amounts to a check that relations $(\mathrm{R} 1-\mathrm{R} 12)$ are preserved by $\Phi$. Now $\mathscr{R}$ contains defining relations for $\mathcal{S B}_{n}$ in $(\mathrm{R} 1-\mathrm{R} 6)$, and for $\mathcal{I B}_{n}$ in $(\mathrm{R} 1-\mathrm{R} 3)$, (R7-R10), and part of (R12); see $[5,7,22]$ and $[16,20]$ respectively. So it remains to check that the equations

$$
\begin{aligned}
\tau_{i} \varepsilon_{j} & =\varepsilon_{j} \tau_{i} & & \text { if } j \neq i, i+1 \\
\tau_{i} \varepsilon_{i}=\sigma_{i} \varepsilon_{i} & =\varepsilon_{i+1} \tau_{i} & & \text { for all } i
\end{aligned}
$$

hold in $\mathcal{P S B}_{n}$. This may be easily accomplished diagrammatically.

The observation in the previous proof that $\mathscr{R}$ contains defining relations for $\mathcal{S B}_{n}$ and $\mathcal{I B}_{n}$ immediately implies the following.

Proposition 13 If $u, v \in\left(S \cup S^{-1} \cup T\right)^{*}$ or $u, v \in\left(S \cup S^{-1} \cup E\right)^{*}$, then $u \Phi=v \Phi$ implies $u \sim v$.

Next we record a technical lemma which will allow us to prove, in Corollary 15 below, a "word version" of the factorization $\mathcal{P} \mathcal{S B}_{n}=\mathcal{E}_{n} \mathcal{S B}_{n}$ from Proposition 1.

Lemma 14 Let $1 \leq i \leq n-1$ and $1 \leq j \leq n$. Then

$$
\begin{aligned}
s_{i}^{ \pm 1} e_{j} \sim \begin{cases}e_{i+1} s_{i}^{ \pm 1} & \text { if } j=i \\
e_{i} s_{i}^{ \pm 1} & \text { if } j=i+1 \\
e_{j} s_{i}^{ \pm 1} & \text { otherwise, }\end{cases} \\
t_{i} e_{j} \sim \begin{cases}e_{i+1} t_{i} & \text { if } j=i \\
e_{i} t_{i} & \text { if } j=i+1 \\
e_{j} t_{i} & \text { otherwise. }\end{cases}
\end{aligned}
$$

Proof Relations (14.1-14.3) follow by Proposition 13 and a simple diagrammatic check, while (14.4) and (14.6) follow immediately from (R12) and (R11) respectively. For (14.5) we have

$$
\begin{aligned}
t_{i} e_{i+1} & \sim s_{i}^{-1} s_{i} t_{i} e_{i+1} & & \text { by }(\mathrm{R} 1) \\
& \sim s_{i}^{-1} t_{i} e_{i} s_{i} & & \text { by }(\mathrm{R} 5) \text { and }(14.2) \\
& \sim s_{i}^{-1} e_{i+1} s_{i} t_{i} & & \text { by (R12) and (R5) } \\
& \sim s_{i}^{-1} s_{i} e_{i} t_{i} & & \text { by (R12) } \\
& \sim e_{i} t_{i} & & \text { by (R1). }
\end{aligned}
$$


For $A \subseteq \mathbf{n}$ with $\mathbf{n} \backslash A=\left\{i_{1}, \ldots, i_{k}\right\}$ and $i_{1}<\cdots<i_{k}$, we define a word

$$
e_{A}=e_{i_{1}} \cdots e_{i_{k}} \in E^{*}
$$

noting that $e_{A} \Phi=\varepsilon_{A}$.

Corollary 15 Let $w \in \mathscr{X}^{*}$ and put $A=\operatorname{dom}(\overline{w \Phi})$. Then $w \sim e_{A} w^{\prime}$ for some word $w^{\prime} \in\left(S \cup S^{-1} \cup T\right)^{*}$.

Proof By repeatedly applying Lemma 14 (if necessary), we have $w \sim w^{\prime \prime} w^{\prime}$ for some $w^{\prime \prime} \in E^{*}$ and $w^{\prime} \in\left(S \cup S^{-1} \cup T\right)^{*}$. By $(\mathrm{R} 7-\mathrm{R} 8)$, we have $w^{\prime \prime} \sim e_{B}$ for some $B \subseteq \mathbf{n}$. But then

$$
B=\operatorname{dom}\left(\operatorname{id}_{B}\right)=\operatorname{dom}\left(\bar{\varepsilon}_{B}\right)=\operatorname{dom}\left(\bar{\varepsilon}_{B} \overline{w^{\prime} \Phi}\right)=\operatorname{dom}\left(\overline{\left(e_{B} w^{\prime}\right) \Phi}\right)=\operatorname{dom}(\overline{w \Phi})=A
$$

since $\overline{w^{\prime} \Phi}$ is a permutation. This completes the proof.

We now define words, for $1 \leq i<j \leq n$, by

$$
\begin{aligned}
a_{i j} & =\left(s_{j-1}^{-1} \cdots s_{i+1}^{-1}\right) s_{i}^{2}\left(s_{i+1} \cdots s_{j-1}\right) \\
g_{i j} & =\left(s_{j-1}^{-1} \cdots s_{i+1}^{-1}\right) s_{i} t_{i}\left(s_{i+1} \cdots s_{j-1}\right),
\end{aligned}
$$

noting that $a_{i j} \Phi=\alpha_{i j}$ and $g_{i j} \Phi=\gamma_{i j}$. We put

$$
U_{n}=\left\{a_{i j}^{ \pm 1}, g_{i j} \mid 1 \leq i<j \leq n\right\} .
$$

Here and elsewhere, if $w=s_{i_{1}}^{ \pm 1} \cdots s_{i_{k}}^{ \pm 1}$ is a word over $S \cup S^{-1}$, we denote by $w^{-1}$ the word $s_{i_{k}}^{\mp 1} \cdots s_{i_{1}}^{\mp 1}$, noting that $w w^{-1} \sim w^{-1} w \sim 1$ for all $w \in\left(S \cup S^{-1}\right)^{*}$.

Lemma 16 Let $w \in \mathscr{X}^{*}$ be such that $\overline{w \Phi}=\operatorname{id}_{A}$ for some $A \subseteq \mathbf{n}$. Then $w \sim e_{A} w^{\prime}$ for some $w^{\prime} \in\left\langle U_{n}\right\rangle$.

Proof Now $w \sim e_{A} w^{\prime \prime}$ for some $w^{\prime \prime} \in\left(S \cup S^{-1} \cup T\right)^{*}$ by Corollary 15. As in the proof of Lemma 11, we fix some factorization

$$
\overline{w^{\prime \prime} \Phi}=\left(i_{1}, j_{1}\right) \cdots\left(i_{k}, j_{k}\right)
$$

of $\overline{w^{\prime \prime} \Phi}$ into transpositions, where $1 \leq i_{r}<j_{r} \leq n$ and $i_{r}, j_{r} \in \mathbf{n} \backslash A$ for each $r \in \mathbf{k}$. Put $w^{\prime \prime \prime}=s_{i_{k} j_{k}} \cdots s_{i_{1} j_{1}}$ where

$$
s_{i j}=\left(s_{j-1}^{-1} \cdots s_{i+1}^{-1}\right) s_{i}\left(s_{i+1} \cdots s_{j-1}\right) .
$$

Then $\varepsilon_{A}\left(w^{\prime \prime \prime} \Phi\right)=\varepsilon_{A}$ in $\mathcal{I B}_{n}$, and it follows that $e_{A} \sim e_{A} w^{\prime \prime \prime}$ by Proposition 13. Now put $w^{\prime \prime \prime \prime}=w^{\prime \prime \prime} w^{\prime \prime}$. Then

$$
\overline{w^{\prime \prime \prime \prime} \Phi}=\overline{w^{\prime \prime \prime} \Phi} \overline{w^{\prime \prime} \Phi}=\left(i_{k}, j_{k}\right) \cdots\left(i_{1}, j_{1}\right)\left(i_{1}, j_{1}\right) \cdots\left(i_{k}, j_{k}\right)=1 .
$$


It follows, by Propositions 9 and 13 , that $w^{\prime \prime \prime \prime} \sim w^{\prime}$ for some $w^{\prime} \in\left\langle U_{n}\right\rangle$, and so

$$
w \sim e_{A} w^{\prime \prime} \sim e_{A} w^{\prime \prime \prime} w^{\prime \prime}=e_{A} w^{\prime \prime \prime \prime} \sim e_{A} w^{\prime},
$$

and the proof is complete.

Lemma 17 Let $1 \leq i<j \leq n$ and $1 \leq k \leq n$. Then

$$
\begin{aligned}
a_{i j}^{ \pm 1} e_{k} & \sim e_{k} a_{i j}^{ \pm 1} \\
g_{i j} e_{k} & \sim e_{k} g_{i j} .
\end{aligned}
$$

Further, if $k \in\{i, j\}$, then

$$
e_{k} a_{i j}^{ \pm 1} \sim e_{k} \sim e_{k} g_{i j}
$$

Proof Relation (17.1) and the first part of (17.3) follow from Proposition 13 and a simple diagrammatic check. For (17.2), first observe that

$$
s_{i} t_{i} e_{\ell} \sim e_{\ell} s_{i} t_{i}
$$

for any $\ell$, by using the two relevant parts of Lemma 14 . We then have

$$
\begin{aligned}
g_{i j} e_{k} & =\left(s_{j-1}^{-1} \cdots s_{i+1}^{-1}\right) s_{i} t_{i}\left(s_{i+1} \cdots s_{j-1}\right) e_{k} & & \\
& \sim\left(s_{j-1}^{-1} \cdots s_{i+1}^{-1}\right) s_{i} t_{i} e_{\ell}\left(s_{i+1} \cdots s_{j-1}\right) & & \text { for some } \ell, \text { by }(14.1-14.3) \\
& \sim\left(s_{j-1}^{-1} \cdots s_{i+1}^{-1}\right) e_{\ell} s_{i} t_{i}\left(s_{i+1} \cdots s_{j-1}\right) & & \text { by the observation } \\
& \sim e_{k}\left(s_{j-1}^{-1} \cdots s_{i+1}^{-1}\right) s_{i} t_{i}\left(s_{i+1} \cdots s_{j-1}\right) & & \text { by }(14.1-14.3) \text { again } \\
& =e_{k} g_{i j} & &
\end{aligned}
$$

establishing (17.2). To complete the proof of (17.3), observe first that

$$
e_{\ell} s_{i} t_{i} \sim e_{\ell} s_{i}^{2} \quad \text { if } \ell \in\{i, i+1\} .
$$

Indeed, we have

$$
e_{i} s_{i} t_{i} \sim s_{i} e_{i+1} t_{i} \sim s_{i} e_{i+1} s_{i} \sim e_{i} s_{i}^{2}
$$

by (14.2) and (R12), while

$$
e_{i+1} s_{i} t_{i} \sim e_{i+1} t_{i} s_{i} \sim e_{i+1} s_{i}^{2}
$$

by (R5) and (R12). It then follows that for $k \in\{i, j\}$ we have

$$
\begin{aligned}
e_{k} g_{i j} & =e_{k}\left(s_{j-1}^{-1} \cdots s_{i+1}^{-1}\right) s_{i} t_{i}\left(s_{i+1} \cdots s_{j-1}\right) & & \\
& \sim\left(s_{j-1}^{-1} \cdots s_{i+1}^{-1}\right) e_{\ell} s_{i} t_{i}\left(s_{i+1} \cdots s_{j-1}\right) & & \text { for some } \ell \in\{i, i+1\}, \text { by }(14.1) \text { or }(14.3) \\
& \sim\left(s_{j-1}^{-1} \cdots s_{i+1}^{-1}\right) e_{\ell} s_{i}^{2}\left(s_{i+1} \cdots s_{j-1}\right) & & \text { by the observation } \\
& \sim e_{k}\left(s_{j-1}^{-1} \cdots s_{i+1}^{-1}\right) s_{i}^{2}\left(s_{i+1} \cdots s_{j-1}\right) & & \text { by }(14.1) \text { or }(14.3) \text { again } \\
& =e_{k} a_{i j}, & &
\end{aligned}
$$


and the proof is complete.

For $A \subseteq \mathbf{n}$ we put

$$
U_{A}=\left\{a_{i j}^{ \pm 1}, g_{i j} \mid 1 \leq i<j \leq n, i, j \in A\right\} .
$$

The next result is an improvement of Lemma 16, and is "almost" a word version of Lemma 11.

Lemma 18 Let $w \in \mathscr{X}^{*}$ be such that $\overline{w \Phi}=\operatorname{id}_{A}$ for some $A \subseteq \mathbf{n}$. Then $w \sim e_{A} w^{\prime}$ for some $w^{\prime} \in\left\langle U_{A}\right\rangle$.

Proof By Lemma 16 we have $w \sim e_{A} w^{\prime \prime}$ for some $w^{\prime \prime} \in\left\langle U_{n}\right\rangle$. Write $w^{\prime \prime}=x_{1} \cdots x_{k}$, where $x_{1}, \ldots, x_{k} \in U_{n}$. If each of $x_{1}, \ldots, x_{k}$ belongs to $U_{A}$ already, then we are done. Otherwise, let $i \in \mathbf{k}$ be minimal so that $x_{i} \in U_{n} \backslash U_{A}$. Then we have $x_{i}=a_{r s}^{ \pm 1}$ or $x_{i}=g_{r s}$ for some $1 \leq r<s \leq n$ such that either $r$ or $s$ belongs to $\mathbf{n} \backslash A$. Let $q \in\{r, s\}$ be such that $q \in \mathbf{n} \backslash A$. We then have

$$
\begin{aligned}
w & \sim e_{A} x_{1} \cdots x_{i-1} x_{i} x_{i+1} \cdots x_{k} & & \\
& \sim e_{A} e_{q} x_{1} \cdots x_{i-1} x_{i} x_{i+1} \cdots x_{k} & & \text { by }(\mathrm{R} 7-\mathrm{R} 8) \\
& \sim e_{A} x_{1} \cdots x_{i-1} e_{q} x_{i} x_{i+1} \cdots x_{k} & & \text { by }(17.1-17.2) \\
& \sim e_{A} x_{1} \cdots x_{i-1} e_{q} x_{i+1} \cdots x_{k} & & \text { by }(17.3) \\
& \sim e_{A} x_{1} \cdots x_{i-1} x_{i+1} \cdots x_{k} & & \text { by }(17.1-17.2) \text { and }(\mathrm{R} 7-\mathrm{R} 8) \text { again. }
\end{aligned}
$$

Since $x_{1} \cdots x_{i-1} x_{i+1} \cdots x_{k}$ contains one fewer factor from $U_{n} \backslash U_{A}$, we are done after applying an induction hypothesis.

We are now ready to prove the main result of this section.

Theorem 19 We have the presentation $\mathcal{P} \mathcal{S B}_{n} \cong\langle\mathscr{X} \mid \mathscr{R}\rangle$.

Proof It remains only to show that $\operatorname{ker} \Phi \subseteq \sim$, so suppose $\left(w_{1}, w_{2}\right) \in \operatorname{ker} \Phi$, and put $A=\operatorname{dom}\left(\overline{w_{1} \Phi}\right)=\operatorname{dom}\left(\overline{w_{2} \Phi}\right)$. Then

$$
w_{1} \sim e_{A} w_{1}^{\prime} \quad \text { and } \quad w_{2} \sim e_{A} w_{2}^{\prime}
$$

for some $w_{1}^{\prime}, w_{2}^{\prime} \in\left(S \cup S^{-1} \cup T\right)^{*}$ by Corollary 15. Choose any $w \in\left(S \cup S^{-1}\right)^{*}$ such that $\overline{w \Phi}=\bar{w}_{1}^{\prime} \Phi$ and put

$$
w_{1}^{\prime \prime}=e_{A} w_{1}^{\prime} w \quad \text { and } \quad w_{2}^{\prime \prime}=e_{A} w_{2}^{\prime} w
$$

Then

$$
w_{1}^{\prime \prime} \Phi=\left(e_{A} w_{1}^{\prime} w\right) \Phi=\left(w_{1} w\right) \Phi=\left(w_{2} w\right) \Phi=\left(e_{A} w_{2}^{\prime} w\right) \Phi=w_{2}^{\prime \prime} \Phi .
$$


This implies that

$$
\overline{w_{2}^{\prime \prime} \Phi}=\overline{w_{1}^{\prime \prime} \Phi}=\overline{e_{A} \Phi} \overline{w_{1}^{\prime} \Phi} \overline{w \Phi}=\mathrm{id}_{A} .
$$

By Lemma 18 we therefore have

$$
w_{1}^{\prime \prime} \sim e_{A} w_{1}^{\prime \prime \prime} \quad \text { and } \quad w_{2}^{\prime \prime} \sim e_{A} w_{2}^{\prime \prime \prime}
$$

for some $w_{1}^{\prime \prime \prime}, w_{2}^{\prime \prime \prime} \in\left\langle U_{A}\right\rangle$. It then follows that

$$
\varepsilon_{A}\left(w_{1}^{\prime \prime \prime} \Phi\right)=w_{1}^{\prime \prime} \Phi=w_{2}^{\prime \prime} \Phi=\varepsilon_{A}\left(w_{2}^{\prime \prime \prime} \Phi\right),
$$

and so $w_{1}^{\prime \prime \prime} \Phi=w_{2}^{\prime \prime \prime} \Phi$ by Lemma 11 , since $w_{1}^{\prime \prime \prime}, w_{2}^{\prime \prime \prime} \in\left\langle\Upsilon_{A}\right\rangle$. But $w_{1}^{\prime \prime \prime}, w_{2}^{\prime \prime \prime} \in\left(S \cup S^{-1} \cup T\right)^{*}$ and so $w_{1}^{\prime \prime \prime} \sim w_{2}^{\prime \prime \prime}$ by Proposition 13. Putting this all together, we have

$$
w_{1} \sim e_{A} w_{1}^{\prime} w w^{-1}=w_{1}^{\prime \prime} w^{-1} \sim e_{A} w_{1}^{\prime \prime \prime} w^{-1} \sim e_{A} w_{2}^{\prime \prime \prime} w^{-1} \sim w_{2}^{\prime \prime} w^{-1}=e_{A} w_{2}^{\prime} w w^{-1} \sim w_{2},
$$

and the proof is complete.

Remark 20 Presentations utilizing the alphabet $S \cup S^{-1} \cup\left\{e_{1}, t_{1}\right\}$ may be derived from $\langle\mathscr{X} \mid \mathscr{R}\rangle$, in view of the equations

$$
\begin{aligned}
\varepsilon_{i} & =\beta_{1 i}^{-1} \varepsilon_{1} \beta_{1 i} \\
\tau_{i} & =\beta_{1 i}^{-1} \beta_{2, i+1}^{-1} \tau_{1} \beta_{2, i+1} \beta_{1 i}
\end{aligned}
$$

where $\beta_{i j}=\sigma_{i} \cdots \sigma_{j-1}$ for each $1 \leq i \leq j \leq n$. Such a presentation would then include the original presentation [12] for $\mathcal{I B}_{n}$, and also a presentation for $\mathcal{S B}_{n}$ in terms of generators $\left\{\sigma_{1}^{ \pm 1}, \ldots, \sigma_{n-1}^{ \pm 1}, \tau_{1}\right\}$; to the author's knowledge, such a presentation for $\mathcal{S B}_{n}$ has not been written down anywhere, although the relations are easy enough to obtain from those in $[5,7,22]$ and share similarities to presentations of the factorizable and permeable braid monoids $\mathfrak{F B}_{n}$ and $\mathfrak{P} \mathcal{B}_{n}$ of $[15,18]$. Of course, the set $\left\{\sigma_{1}^{ \pm 1}, \ldots, \sigma_{n-1}^{ \pm 1}\right\}$ is not a minimal generating set for the braid group $\mathcal{B}_{n}$, as these $2(n-1)$ generators may be replaced by the three braids $\sigma_{1}, \sigma_{2} \cdots \sigma_{n-1}, \sigma_{n-1}^{-1} \cdots \sigma_{1}^{-1}$. The resulting five-element generating set for $\mathcal{P S B}_{n}$ is certainly minimal, but it is the opinion of the author that a set of defining relations with respect to these generators would not be "natural" enough to warrant investigation.

\section{The Desingularization Map}

The primary reason for the introduction of the singular braid monoid $\mathcal{S B}_{n}$ in $[5,7]$ was its connection to Vassiliev invariants of knots and links; see also [28]. The exact connection lies in the existence of a homomorphism from $\mathcal{S B}_{n}$ into the complex group algebra $\mathbb{C}\left[\mathcal{B}_{n}\right]$ of the braid group induced by $\sigma_{i}^{ \pm 1} \mapsto \sigma_{i}^{ \pm 1}$ and $\tau_{i} \mapsto \sigma_{i}-\sigma_{i}^{-1}$. It was conjectured in [7], and proved in [27], that this homomorphism - the so-called Vassiliev homomorphism, or desigularization map - is injective. In this section we derive a similar result for the partial 
singular braid monoid $\mathcal{P S B}_{n}$; specifically, we show that there is a natural desingularization map from $\mathcal{P S} \mathcal{S}_{n}$ into the complex semigroup algebra $\mathbb{C}\left[\mathcal{I} \mathcal{B}_{n}\right]$ of the inverse braid monoid $\mathcal{I} \mathcal{B}_{n}$, and we show that this map is injective.

We begin by defining a map

$$
\psi: \mathscr{X}^{*} \rightarrow \mathbb{C}\left[\mathcal{I B}_{n}\right]:\left\{\begin{aligned}
s_{i}^{ \pm 1} & \mapsto \sigma_{i}^{ \pm 1} \\
e_{i} & \mapsto \varepsilon_{i} \\
t_{i} & \mapsto \frac{1}{2}\left(\sigma_{i}+\sigma_{i}^{-1}\right)
\end{aligned}\right.
$$

A simple check of the relations shows that $\sim \subseteq$ ker $\psi$, so that $\psi$ induces a homomorphism $\mathscr{X}^{*} / \sim \rightarrow \mathbb{C}\left[\mathcal{I B}_{n}\right]$. By Theorem 19 it follows that we have a well-defined homomorphism

$$
\Psi: \mathcal{P S} \mathcal{B}_{n} \rightarrow \mathbb{C}\left[\mathcal{I B}_{n}\right]
$$

under which

$$
\sigma_{i}^{ \pm 1} \Psi=\sigma_{i}^{ \pm 1}, \quad \varepsilon_{i} \Psi=\varepsilon_{i}, \quad \tau_{i} \Psi=\frac{1}{2}\left(\sigma_{i}+\sigma_{i}^{-1}\right) .
$$

As alluded to above, it is our goal in this section to show that $\Psi$ is injective.

Remark 21 The exact form of $t_{i} \psi=\frac{1}{2}\left(\sigma_{i}+\sigma_{i}^{-1}\right)$ was chosen with relation (R12) in mind. If we had instead chosen to define $t_{i} \psi=\sigma_{i}-\sigma_{i}^{-1}$ as in [7], or $t_{i} \psi=\sigma_{i}+\sigma_{i}^{-1}$ as in [24], then $\left(t_{i} e_{i}\right) \psi$ would have been equal to 0 , or $2 \sigma_{i} \varepsilon_{i}$, respectively, in either case not preserving (R12). If we instead define $t_{i} \psi=a_{i} \sigma_{i}+b_{i} \sigma_{i}^{-1}$ for some collection of scalars $a_{i}, b_{i} \in \mathbb{C}$ and want $\sim \subseteq$ ker $\psi$, then (R6) forces $a_{i}=a_{1}$ and $b_{i}=b_{1}$ for all $i$, while (R12) forces $a_{1}+b_{1}=1$. If $a_{1}=0$ or $b_{1}=0$, then the induced map $\Psi: \mathcal{P} \mathcal{S} \mathcal{B}_{n} \rightarrow \mathbb{C}\left[\mathcal{I} \mathcal{B}_{n}\right]$ would not be injective, since then $\sigma_{i}^{-1} \Psi=\tau_{i} \Psi=\sigma_{i}^{-1}$ or $\sigma_{i} \Psi=\tau_{i} \Psi=\sigma_{i}$ respectively. The arguments we use throughout this section may easily be adapted to treat the more general map defined with non-zero $a_{1}, b_{1}$ satisfying $a_{1}+b_{1}=1$ as above.

Recall that for $0 \leq k \leq n$, the singular braid monoid $\mathcal{S B}_{k}$ is contained in $\mathcal{P} \mathcal{S B}_{n}$ as the set of all $\beta \in \mathcal{P S B}_{n}$ satisfying $\operatorname{dom}(\bar{\beta})=\operatorname{im}(\bar{\beta})=\mathbf{k}$. We will denote by $\sigma_{i ; k}^{ \pm 1}, \tau_{i ; k}(i=1, \ldots, k-1)$ the canonical generators of $\mathcal{S B}_{k}$. Note that we have

$$
\sigma_{i ; k}^{ \pm 1}=\varepsilon_{\mathbf{k}} \sigma_{i}^{ \pm 1} \quad \text { and } \quad \tau_{i ; k}=\varepsilon_{\mathbf{k}} \tau_{i}
$$

for each $i$. By [7] and [24], for each $0 \leq k \leq n$, there exist homomorphisms

$$
\begin{aligned}
\eta_{k}: \mathcal{S B}_{k} & \rightarrow \mathbb{C}\left[\mathcal{B}_{k}\right]: \sigma_{i ; k}^{ \pm 1} \mapsto \sigma_{i ; k}^{ \pm 1}, \tau_{i ; k} \mapsto \sigma_{i ; k}-\sigma_{i ; k}^{-1} \\
\zeta_{k}: \mathcal{S B}_{k} & \rightarrow \mathbb{C}\left[\mathcal{B}_{k}\right]: \sigma_{i ; k}^{ \pm 1} \mapsto \sigma_{i ; k}^{ \pm 1}, \tau_{i ; k} \mapsto \sigma_{i ; k}+\sigma_{i ; k}^{-1}
\end{aligned}
$$

In [24] it was shown that injectivity of $\eta_{k}$ is equivalent to injectivity of $\zeta_{k}$ and, in [27], the injectivity of $\eta_{k}$ (and hence also of $\zeta_{k}$ ) was demonstrated. To simplify subsequent proofs, it will be convenient to record the next intermediate result. 
Lemma 22 For all $0 \leq k \leq n$, the map

$$
\xi_{k}: \mathcal{S B}_{k} \rightarrow \mathbb{C}\left[\mathcal{B}_{k}\right]: \beta \mapsto \frac{1}{2^{N(\beta)}}\left(\beta \zeta_{k}\right)
$$

is an injective homomorphism.

Proof Using the rule $N(\beta \gamma)=N(\beta)+N(\gamma)$ for all $\beta, \gamma \in \mathcal{S B}_{k}$, and the fact that $\zeta_{k}$ is a homomorphism, we quickly deduce that $\xi_{k}$ is a homomorphism. Suppose now that $\beta, \gamma \in \mathcal{S B}_{k}$ are such that $\beta \xi_{k}=\gamma \xi_{k}$. Now

$$
\beta \xi_{k}=\sum_{\delta \in \mathcal{B}_{k}} c_{\delta} \delta
$$

where only finitely many of the coefficients $c_{\delta} \in \mathbb{C}$ are non-zero. Put $\Xi=\left\{\delta \in \mathcal{B}_{k} \mid c_{\delta} \neq 0\right\}$. By definition of $\zeta_{k}$, there is a unique braid $\delta_{*} \in \Xi$ of maximal exponent sum (the exponent sum of a braid $\delta \in \mathcal{B}_{k}$ is $r_{1}+\cdots+r_{\ell}$ for any expression $\delta=\sigma_{i_{1} ; k}^{r_{1}} \cdots \sigma_{i_{\ell} ; k}^{r_{\ell}}$ ), and we clearly have $c_{\delta_{*}}=\frac{1}{2^{N(\beta)}}$. Since $\beta \xi_{k}=\gamma \xi_{k}$, it follows also that $c_{\delta_{*}}=\frac{1}{2^{N(\gamma)}}$ and so $N(\beta)=N(\gamma)$. But then

$$
\beta \zeta_{k}=2^{N(\beta)}\left(\beta \xi_{k}\right)=2^{N(\gamma)}\left(\gamma \xi_{k}\right)=\gamma \zeta_{k},
$$

so that $\beta=\gamma$, by the injectivity of $\zeta_{k}$. This completes the proof.

Lemma 23 For all $0 \leq k \leq n$, the restriction $\left.\Psi\right|_{\mathcal{S B}_{k}}$ is injective.

Proof If $k=0,1$ then $\mathcal{S B}_{k}$ has only one element and the result is trivial, so suppose $k \geq 2$. One easily checks the formulae

$$
\sigma_{i ; k}^{ \pm 1} \Psi=\sigma_{i ; k}^{ \pm 1} \quad \text { and } \quad \tau_{i ; k} \Psi=\frac{1}{2}\left(\sigma_{i ; k}+\sigma_{i ; k}^{-1}\right) .
$$

So $\Psi$ and $\xi_{k}$ agree on the generators of $\mathcal{S B}_{k}$ and it follows that $\left.\Psi\right|_{\mathcal{S B}_{k}}=\xi_{k}$, and we are done by the previous lemma.

We have now come to the main result of this section.

Theorem 24 The desingularization map $\Psi: \mathcal{P} \mathcal{S B}_{n} \rightarrow \mathbb{C}\left[\mathcal{I B}_{n}\right]$ is injective.

Proof Suppose $\beta, \gamma \in \mathcal{P} \mathcal{S B}_{n}$ are such that $\beta \Psi=\gamma \Psi$. Write

$$
\beta \Psi=\sum_{\delta \in \mathcal{I B}_{n}} c_{\delta} \delta
$$

where each $c_{\delta} \in \mathbb{C}$, and put $\Xi=\left\{\delta \in \mathcal{I} \mathcal{B}_{n} \mid c_{\delta} \neq 0\right\}$. We have $\bar{\delta}=\bar{\beta}$ for all $\delta \in \Xi$, by the definition of $\psi$. It follows that $\bar{\beta}=\bar{\gamma}$. Put $A=\operatorname{dom}(\bar{\beta}), B=\operatorname{im}(\bar{\beta})$, and $k=|\beta|$. Let $\widehat{\beta}, \widehat{\gamma} \in \mathcal{S B}_{k}$ be as in Lemma 4 . We then have

$$
\widehat{\beta} \Psi=\left(\rho_{A} \beta \lambda_{B}\right) \Psi=\left(\rho_{A} \Psi\right)(\beta \Psi)\left(\lambda_{B} \Psi\right)=\left(\rho_{A} \Psi\right)(\gamma \Psi)\left(\lambda_{B} \Psi\right)=\left(\rho_{A} \gamma \lambda_{B}\right) \Psi=\widehat{\gamma} \Psi,
$$


so that $\widehat{\beta}=\widehat{\gamma}$ by Lemma 23. But then

$$
\beta=\lambda_{A} \widehat{\beta} \rho_{B}=\lambda_{A} \widehat{\gamma} \rho_{B}=\gamma
$$

and the proof is complete.

\section{References}

[1] J. W. Alexander. A Lemma on Systems of Knotted Curves. Proc. Nat. Acad. Sci. U.S.A., 9:93-95, 1923.

[2] N. Antony. On Singular Artin Monoids and Contributions to Birman's Conjecture. Comm. Algebra, 33(11):4043-4056, 2005.

[3] E. Artin. Theorie der Zöpfe. Abh. Math. Sem. Univ. Hamburg, 4:47-72, 1925.

[4] E. Artin. Theory of Braids. Ann. of Math., 48(2):101-126, 1947.

[5] J. C. Baez. Link Invariants of Finite Type and Perturbation Theory. Lett. Math. Phys., 26(1):43-51, 1992.

[6] J. S. Birman. Braids, Links, and Mapping Class Groups, volume 82 of Annals of Mathematical Studies. Princeton University Press, Princeton, New Jersey, 1975.

[7] J. S. Birman. New Points of View in Knot Theory. Bull. Amer. Math. Soc. (N.S.), 28(2):253-287, 1993.

[8] J. Cockett and S. Lack. Restriction Categories. I. Categories of Partial Maps. Theoret. Comput. Sci., 270(1-2):223-259, 2002.

[9] R. Corran. A Normal Form for a Class of Monoids Including the Singular Braid Monoids. J. Algebra, 223(1):256-282, 2000.

[10] R. Corran. Conjugacy in Singular Artin Monoids. J. Aust. Math. Soc., 79(2):183-212, 2005.

[11] D. Easdown, J. East, and D. G. FitzGerald. Braids and Factorizable Inverse Monoids. Semigroups and Languages, eds. I.M. Araújo, M.J.J. Branco, V.H. Fernandes, and G.M.S. Gomes, World Scientific, pages 86-105, 2002.

[12] D. Easdown and T. G. Lavers. The Inverse Braid Monoid. Adv. Math., 186(2):438-455, 2004.

[13] J. East. Birman's Conjecture is True for $I_{2}(p)$. J. Knot Theory Ramifications, 15(2):167-177, 2006. 
[14] J. East. Braids and Order-Preserving Partial Permutations. Preprint, 2006.

[15] J. East. The Factorizable Braid Monoid. Proc. Edinb. Math. Soc. (2), 49(3):609-636, 2006.

[16] J. East. Braids and Partial Permutations. Adv. Math., 213(1):440-461, 2007.

[17] J. East. Vines and Partial Transformations. Adv. Math., 216(2):787-810, 2007.

[18] J. East. On a Class of Factorizable Inverse Monoids associated with Braid Groups. Comm. Algebra, 36(8):3155-3190, 2008.

[19] Vitor H. Fernandes. The Monoid of all Injective Order Preserving Partial Transformations on a Finite Chain. Semigroup Forum, 62(2):178-204, 2001.

[20] N. D. Gilbert. Presentations of the Inverse Braid Monoid. J. Knot Theory Ramifications, 15(5):571-588, 2006.

[21] E. Godelle and L. Paris. On Singular Artin Monoids. Procedings of Geometric Methods in Group Theory (Contemp. Math., 372, Amer. Math. Soc., Providence, RI), pages 43-57, 2005.

[22] J. González-Meneses. Presentations for the Monoids of Singular Braids on Closed Surfaces. Comm. Algebra, 30(6):2829-2836, 2002.

[23] J. Green. On the Structure of Semigroups. Ann. of Math. (2), 54:163-172, 1951.

[24] Antal Járai Jr. On the Monoid of Singular Braids. Topology Appl., 96:109-119, 1999.

[25] T. G. Lavers. The Theory of Vines. Comm. Algebra, 25(4):1257-1284, 1997.

[26] L. Paris. Birman's Conjecture for Singular Braids on Closed Surfaces. J. Knot Theory Ramifications, 13(7):895-915, 2004.

[27] L. Paris. The Proof of Birman's Conjecture on Singular Braid Monoids. Geom. Topol., 8:1281-1300, 2004.

[28] V. Vassiliev. Cohomology of Knot Spaces. Theory of singularities and its applications (Ed. V. Arnold), Adv. Soviet Math., 1, Amer. Math. Soc., Providence, RI, pages 23-69, 1990. 